\title{
Carleson Measure of Harmonic Schwarzian Derivatives Associated with a Finitely Generated Fuchsian Group of the Second Kind
}

\author{
Guangming Hu, ${ }^{1}$ Yutong Liu $\mathbb{D}^{2}{ }^{2}$ Yu Sun, ${ }^{3}$ and Xinjie Qian ${ }^{1}$ \\ ${ }^{1}$ College of Science, Jinling Institute of Technology, Nanjing 211169, China \\ ${ }^{2}$ School of Mathematics and Systems Science, Beihang University, Beijing 100191, China \\ ${ }^{3}$ School of Mathematical Sciences, Yangzhou University, Yangzhou 225001, China \\ Correspondence should be addressed to Yutong Liu; 974889738@qq.com
}

Received 10 February 2021; Revised 3 March 2021; Accepted 13 March 2021; Published 22 March 2021

Academic Editor: Dumitru Motreanu

Copyright ( 92021 Guangming Hu et al. This is an open access article distributed under the Creative Commons Attribution License, which permits unrestricted use, distribution, and reproduction in any medium, provided the original work is properly cited.

Let $S_{\mathrm{H}}(f)$ be the Schwarzian derivative of a univalent harmonic function $f$ in the unit disk $\mathbb{D}$, compatible with a finitely generated Fuchsian group $G$ of the second kind. We show that if $\left|S_{\mathrm{H}}(f)\right|^{2}\left(1-|z|^{2}\right)^{3} d x d y$ satisfies the Carleson condition on the infinite boundary of the Dirichlet fundamental domain $\mathscr{F}$ of $G$, then $\left|S_{\mathrm{H}}(f)\right|^{2}\left(1-|z|^{2}\right)^{3} d x d y$ is a Carleson measure in $\mathbb{D}$.

\section{Introduction}

Throughout this paper, we adopt the conventional symbols, $\mathbb{D}=\{z:|z|<1\}$ and $B(z, r)$, to denote the unit disk in the extended complex plane $\widehat{\mathbb{C}}$ and the disk with center $z$ and radius $r$, respectively. Moreover, use $S^{1}=\partial \mathbb{D}$ to denote the boundary of $\mathbb{D}$.

A complex-valued function $f$ is said to be complexvalued harmonic in $\mathbb{D}$ if the real part and the imaginary part of $f$ are real harmonic in $\mathbb{D}$. Notice that every complexvalued harmonic function $f$ can be written as $f=h+\bar{g}$, where both $g$ and $h$ are analytic in $\mathbb{D}$. Moreover, a complex-valued harmonic function $f$ is called to be locally univalent if its Jacobian determinant

$$
J_{f}=\left|f_{z}\right|^{2}-\left|f_{\bar{z}}\right|^{2}=\left|h^{\prime}\right|^{2}-\left|g^{\prime}\right|^{2}
$$

does not vanish in $\mathbb{D}$. It follows that if $f$ is locally univalent, then $f$ is either sense-preserving or sense-reversing depending on the conditions $J_{f}>0$ and $J_{f}<0$ in $\mathbb{D}$, respectively.

Notice that if $f$ is sense-preserving, then $\bar{f}$ is sensereversing. Let $\omega=g^{\prime} / h^{\prime}$ be the (second complex) dilatation of $f=h+\bar{g}$. It follows from [1] that, if a locally univalent har- monic function $f=h+\bar{g}$ is sense-preserving, then its analytic part $h$ is locally univalent and $\omega=g^{\prime} / h^{\prime}$ is analytic with $|\omega|$ $<1$. Moreover, a locally univalent harmonic function $f$ is univalent if $f$ is injective.

Recall that the harmonic pre-Schwarzian derivative $P_{\mathrm{H}}$ of a locally univalent harmonic function $f$ with $J_{f}$ is defined by

$$
P_{\mathrm{H}}(f)=\frac{\partial}{\partial z} \log J_{f}=\frac{h^{\prime \prime}}{h^{\prime}}-\frac{\bar{\omega} \omega^{\prime}}{1-|\omega|^{2}},
$$

and the harmonic Schwarzian derivative $S_{\mathrm{H}}[2-4]$ is defined as

$$
\begin{aligned}
S_{\mathrm{H}}(f)= & \frac{\partial}{\partial z} P_{\mathrm{H}}(f)-\frac{1}{2} P_{\mathrm{H}}^{2}(f)=S(h)+\frac{\bar{\omega}}{1-|\omega|^{2}}\left(\frac{h^{\prime \prime} \omega^{\prime}}{h^{\prime}}-\omega^{\prime \prime}\right) \\
& -\frac{3}{2}\left(\frac{\bar{\omega} \omega^{\prime}}{1-|\omega|^{2}}\right)^{2} .
\end{aligned}
$$

Since $P_{\mathrm{H}}(f)=(\partial / \partial z) \log J_{f}$, we can obtain that $P_{\mathrm{H}}(f)=$ $P_{\mathrm{H}}(\bar{f})$, where $f$ is harmonic and sense-preserving. It implies 
that both the pre-Schwarzian derivative and the Schwarzian derivative are well-defined for the locally univalent harmonic function (sense-preserving or sense-reversing) in $\mathbb{D}$.

Now, we shall introduce some basic concepts concerning the Fuchsian group. Firstly, a Möbius transformation of $\mathbb{D}$ is defined by

$$
A(z)=e^{i \theta} \frac{z-a}{1-\bar{a} z}, a \in \mathbb{D}, \theta \in[0,2 \pi) .
$$

A Fuchsian group is a discrete Möbius group $G$ acting on the unit disk $\mathbb{D}$. For a Fuchsian group $G$, it is cocompact if $\mathbb{D} / G$ is compact and is convex cocompact if $G$ is finitely generated without parabolic elements. Furthermore, a Fuchsian group $G$ is of the first kind if its limit set is $S^{1}$; otherwise, it is of the second kind. Notice that all cocompact groups are the first kind and convex cocompact groups minus cocompact groups are the second kind.

A finitely generated Fuchsian group $G$ is called to be of divergence type if

$$
\sum_{g \in G}(1-|g(0)|)=\infty o r \sum_{g \in G} \exp (-\rho(0, g(0)))=\infty
$$

and to be of convergence type otherwise, where $\rho(\cdot, \cdot)$ is the hyperbolic distance. We know that all finitely generated Fuchsian groups of the second kind are of convergence type. For more details about Fuchsian groups, see [5].

On the basis of the above definitions, we call a locally univalent harmonic function $f$ compatible with a Fuchsian group $G$ if and only if $f \circ A=f$, for any $A \in G$. Correspondingly, the Schwarzian derivative $S_{\mathrm{H}}(f)$ is called a $G$-compatible Schwarzian derivative if $f$ is a $G$-compatible locally univalent harmonic function. Since

$$
S_{\mathrm{H}}(f \circ A)=\left(S_{\mathrm{H}}(f) \circ A\right) \cdot\left(A^{\prime}\right)^{2}
$$

then a $G$-compatible Schwarzian derivative $S_{\mathrm{H}}(f)$ should satisfy

$$
S_{\mathrm{H}}(f)=\left(S_{\mathrm{H}}(f) \circ A\right) \cdot\left(A^{\prime}\right)^{2}, A \in G
$$

A positive measure $\lambda$, defined on a simply connected domain $\Omega$, is called a Carleson measure if there exists a positive constant $C$, independent of $r$, such that for all $0<r$ $<\operatorname{diameter}(\partial \Omega)$ and $z \in \partial \Omega$,

$$
\lambda(\Omega \cap B(z, r)) \leq C r .
$$

The Carleson norm $\|\lambda\|_{\Omega}$ of $\lambda$ is defined by

$\|\lambda\|_{\Omega}=\sup \left\{\frac{\lambda(\Omega \cap B(z, r))}{r}: z \in \partial \Omega, 0<r<\operatorname{diameter}(\Omega)\right\}<\infty$.
Denote by $\operatorname{CM}(\Omega)$ the set of all Carleson measures on $\Omega$. Correspondingly, CM $(\mathbb{D})$ is the set of all Carleson measures on $\mathbb{D}$. For more details, see [6].

Let $\mathscr{F}$ be the Dirichlet fundamental domain of a Fuchsian group $G$ in $\mathbb{D}$ centered at $z=0$ and $\mathscr{F}(\infty)$ be the boundary at infinity of $\mathscr{F}$. Huo [7] considered a Beltrami coefficient $\mu$ in $\mathbb{D}$ compatible with a finitely generated Fuchsian group $G$ of the second kind and showed that if $\left(|\mu| /\left(1-|z|^{2}\right) 1-|z|^{2}\right) d x$ $d y$ satisfies the Carleson condition on $\mathscr{F}(\infty)$, then $(|\mu| /(1$ $\left.\left.-|z|^{2}\right) 1-|z|^{2}\right) d x d y$ is a Carleson measure in $\mathbb{D}$. Naturally, one may ask whether it is right for the Schwarzian derivative $S_{\mathrm{H}}(f)$ of a $G$-compatible locally univalent harmonic function or not. For the case of a $G$-compatible univalent harmonic function, the following theorem will give an affirmation of the above problem.

Theorem 1. Let $G$ be any finitely generated Fuchsian group of the second kind and $\mathscr{F}$ be the Dirichlet fundamental domain of $G$ centered at 0 . For a $G$-compatible univalent harmonic function $f$, if there exists a constant $C>0$ such that, for any $\xi \in \mathscr{F}(\infty)$ and any $0<r<2$,

$$
\iint_{B(\xi, r)}\left|S_{H}(f)\right|^{2}\left(1-|z|^{2}\right)^{3} \chi_{\mathscr{F}} d x d y \leq C r
$$

then $\left|S_{H}(f)\right|^{2}\left(1-|z|^{2}\right)^{3} d x d y$ is in $C M(\mathbb{D})$, where $\chi_{\mathscr{F}}$ is the characteristic function of $\mathscr{F}$.

The rest of this paper is organized as follows. In Section 2, we give some related lemmas. In Section 3, we divide two parts to give the proof of Theorem 1 .

\section{Some Lemmas}

The following lemma is used several times in this paper, and we shall give a short proof in this section.

Lemma 2. Suppose that $f$ is a univalent harmonic function in D. If

$$
\left|S_{H}(f)\right|^{2}\left(1-|z|^{2}\right)^{3} d x d y \in C M(\mathbb{D})
$$

then there exists a constant $C>0$ such that, for any $\xi \in \overline{\mathbb{D}}$ and $0<r<2$,

$$
\iint_{B(\xi, r) \cap \mathbb{D}}\left|S_{H}(f)\right|^{2}\left(1-|z|^{2}\right)^{3} d x d y \leq C r
$$

where $C$ depends only on the Carleson norm of $\left|S_{H}(f)\right|^{2}$ $\left(1-|z|^{2}\right)^{3} d x d y$.

Proof. Choose $0<r<2$ and fix it. For any $\xi \in \overline{\mathbb{D}}$, if $\xi \in \partial \mathbb{D}$, it is obviously right. For $\xi \in \mathbb{D}$, if the Euclidean distance $\operatorname{dist}(\xi, \partial$ $\mathbb{D}) \geq 2 r$ (this case only happens when $0<r<1 / 2$ ), then, by 
Theorem 5 of [1], we have

$$
\begin{aligned}
\iint_{B(\xi, r)}\left|S_{\mathrm{H}}(f)\right|^{2}\left(1-|z|^{2}\right)^{3} d x d y & \leq \iint_{B(\xi, r)} \frac{C_{1}}{1-|z|^{2}} d x d y \\
& \leq \frac{C_{1} \pi r^{2}}{1-|1-r|^{2}}=\frac{C_{1} \pi r}{2-r} \leq C_{2} r,
\end{aligned}
$$

where $C_{1}$ and $C_{2}$ are universal positive constants.

In the case of $\operatorname{dist}(\xi, \partial \mathbb{D})<2 r$, we can choose a point $\eta$ $\in \partial \mathbb{D}$ such that $\operatorname{dist}(\eta, \xi)<2 r$. Then, we have $B(\xi, r) \subset B(\eta$, $4 r)$ and

$$
\begin{aligned}
\iint_{B(\xi, r) \cap \mathbb{D}}\left|S_{\mathrm{H}}(f)\right|^{2}\left(1-|z|^{2}\right)^{3} d x d y & \leq \iint_{B(\eta, 4 r) \cap \mathbb{D}}\left|S_{\mathrm{H}}(f)\right|^{2}\left(1-|z|^{2}\right)^{3} d x d y \\
& \leq 4 C^{*} r,
\end{aligned}
$$

where $C^{*}$ is the Carleson norm of the measure $\left|S_{\mathrm{H}}(f)\right|^{2}$ $\left(1-|z|^{2}\right)^{3} d x d y$.

Therefore, set $C=\max \left\{C_{2}, 4 C^{*}\right\}$ and the proof of this lemma is complete.

By the above lemma, we know that for any simply connected domain $\Omega \subset \mathbb{D}$, if $\left|S_{\mathrm{H}}(f)\right|^{2}\left(1-|z|^{2}\right)^{3} d x d y$ is a Carleson measure on $\mathbb{D}$, then it is also a Carleson measure on $\Omega$.

Lemma 3. Let $G$ be a convergence-type Fuchsian group and $f$ be a $G$-compatible univalent harmonic function. If there exists $0<t<1$ such that the support set of $S_{H}(f) \chi_{\mathscr{F}}$ is contained in $B(0, t)$, then $\left|S_{H}(f)\right|^{2}\left(1-|z|^{2}\right)^{3} d x d y \in C M(\mathbb{D})$.

Proof. Suppose that the support set of $S_{\mathrm{H}}(f) \chi_{\mathscr{F}}$, denoted by $\operatorname{Supp}\left(S_{\mathrm{H}}(f)\right)$, is contained in $B(0, t)$. Recall that a sequence $\left\{z_{j}\right\}$ is called an interpolating sequence of $\mathbb{D}$, if it satisfies the following two conditions:

$$
\begin{gathered}
\exists \eta>0, \rho\left(z_{j}, z_{k}\right) \geq \eta \quad \text { for } j \neq k, \\
\sum\left(1-\left|z_{i}\right|^{2}\right) \delta_{z_{i}} \in \mathrm{CM}(\mathbb{D}),
\end{gathered}
$$

where $\delta_{z}$ stands for the Dirac mass at $z$.

Huo [7] has shown that the sequence $\{g(0)\}_{g \in G}$ is an interpolating sequence in $\mathbb{D}$. Hence, for any $\xi \in \partial \mathbb{D}$ and $0<$ $r<2$, by Theorem 5 of [1], we have

$$
\begin{aligned}
L & =\iint_{\mathbb{D} \cap B(\xi, r)}\left|S_{\mathrm{H}}(f)\right|^{2}\left(1-|z|^{2}\right)^{3} d x d y \\
& \leq \sum_{g \in G} \iint_{g(B(0, t)) \cap B(\xi, r)}\left|S_{\mathrm{H}}(f)\right|^{2}\left(1-|z|^{2}\right)^{3} d x d y \\
& =\sum_{g \in G} \iint_{g(B(0, t))}\left|S_{\mathrm{H}}(f)\right|^{2}\left(1-|z|^{2}\right)^{3} \chi_{B(\xi, r)} d x d y \\
& \leq \sum_{g \in G} \iint_{g(B(0, t))} \frac{C}{1-|z|^{2}} \chi_{B(\xi, r)} d x d y,
\end{aligned}
$$

where $C$ is a universal positive constant.
Note that the hyperbolic radius $t_{\rho}$ of the Euclidean disk $B(0, t)$ is $\ln ((1+t) 1+t /(1-t) 1-t)$. Therefore, for any $g \in$ $G$, the disk $g(B(0, t))$ is a hyperbolic disk with center $g(0)$ and hyperbolic radius $t_{\rho}$. By [8], we know that $g(B(0, t))$ is contained in the Euclidean disk $B\left(g(0), R_{g}\right)$ and $R_{g} \leq C_{3}(1$ $-|g(0)|)$, where $C_{3}$ is a constant depending on $t$.

Combined with the above discussion, we have

$$
L \leq \sum_{g(0) \in B(\xi, r)} \frac{C \pi R_{g}^{2}}{1-\left|1-R_{g}\right|^{2}} \leq C_{4} \sum_{g(0) \in B(\xi, r)}(1-|g(0)|) \leq C^{*} r,
$$

where $C^{*}$ depends on $C_{3}, C_{4}$, and the Carleson norm of the measure $\sum_{g \in G}(1-|g(0)|) \delta_{g(0)}$.

Lemma 4 [9]. Let $\Omega$ be a chord-arc domain. Then, the following statements are equivalent:

(a) $d \mu$ is a Carleson measure in $\Omega$

(b) For $0<p<\infty, f \in H^{p}(\Omega)$, we have

$$
\iint_{\Omega}|f|^{p} d \mu \leq C \int_{\partial \Omega}|f|^{p} d s
$$

where $H^{p}(\Omega)=\left\{f: f\right.$ is analytic in $\Omega$ and $\left.\int_{\partial \Omega}|f|^{p} d s<\infty\right\}$ and $C$ only depends on the Carleson norm of $d \mu$.

\section{Proof of Theorem 1}

In order to prove Theorem 1, we divide two parts for the finitely generated Fuchsian group of the second kind $G$ as follows: the first case is to consider the finitely generated Fuchsian group of the second kind $G$ without any parabolic element; the second case is to discuss the finitely generated Fuchsian group of the second kind $G$ with some parabolic elements.

Theorem 5. Let $G$ be a finitely generated Fuchsian group of the second kind without any parabolic element and $\mathscr{F}$ be the Dirichlet fundamental domain of $G$ centered at 0 . For a $G$ -compatible univalent harmonic function $f$, if there exists a constant $C>0$ such that, for any $\xi \in \mathscr{F}(\infty)$ and any $0<r<2$,

$$
\iint_{B(\xi, r)}\left|S_{H}(f)\right|^{2}\left(1-|z|^{2}\right)^{3} \chi_{\mathscr{F}} d x d y \leq C r,
$$

then $\left|S_{H}(f)\right|^{2}\left(1-|z|^{2}\right)^{3} d x d y$ is in $C M(\mathbb{D})$, where $\chi_{\mathscr{F}}$ is the characteristic function of the Dirichlet fundamental domain $\mathscr{F}$.

Proof. Let $G$ be a finitely generated Fuchsian group of the second kind without any parabolic element and $\mathscr{F}$ be the Dirichlet domain of $G$ with center 0 . Let $f$ be a $G$-compatible univalent harmonic function. Then, the intersection of the closure of $\mathscr{F}$ with $\partial \mathbb{D}$ contains finitely many intervals which are called free edges of $\mathscr{F}$, denoted by $I_{1}, I_{2}, \cdots, I_{n}$. 
For any $1 \leq i \leq n$, let $q_{i, 1}, q_{i, 2}$ be the endpoints of $I_{i}$. It is known that both $q_{i, 1}, q_{i, 2}$ do not belong to the limit set. Both sides of $q_{i, j}(j=1$, or 2$)$ are free sides of Dirichlet fundamental domains with different centers.

By the statement of the theorem, there exists a constant $C>0$ such that, for $1 \leq i \leq n$, choose a ball $B_{i}$ such that $B_{i} \cap$ $\partial \mathbb{D}$ contains no limit points of $G$, and $I_{i} \subset B_{i} \cap \partial \mathbb{D}$. Then, for any point $\xi \in I_{i}, 0<r<2$, we have

$$
\iint_{B(\xi, r) \cap \mathbb{D}}\left|S_{\mathrm{H}}(f)\right|^{2}\left(1-|z|^{2}\right)^{3} \chi_{B_{i} \cap \mathbb{D}} d x d y \leq C r .
$$

Furthermore, the set $\mathscr{F}_{c}=\overline{\mathscr{F}}-\bigcup_{i=1}^{n}\left(B_{i} \cap \mathscr{F}\right)$ is compact, where $\overline{\mathscr{F}}$ is the closure of $\mathscr{F}$.

By Lemma 2, we know that $\left|S_{\mathrm{H}}(f)\right|^{2}\left(1-|z|^{2}\right)^{3} d x d y$ is a Carleson measure in $B_{i} \cap \mathscr{F}$.

We shall now divide $f$ into two parts $f_{1}, f_{2}$,

$$
f_{1}=\sum_{g \in G} f \chi_{g\left(\mathscr{F}_{c}\right)}, f_{2}=\sum_{g \in G} f \chi_{g(B)},
$$

where $B=\bigcup_{i=1}^{n}\left(B_{i} \cap \mathscr{F}\right)$. By Lemma 3 , we know that the measure $\left|S_{\mathrm{H}}\left(f_{1}\right)\right|^{2}\left(1-|z|^{2}\right)^{3} d x d y$ is a Carleson measure on $\mathbb{D}$. Next, we only need to show that $\left|S_{\mathrm{H}}\left(f_{2}\right)\right|^{2}\left(1-|z|^{2}\right)^{3} d x d y$ is also a Carleson measure.

Let $\xi$ be an arbitrary point on $\partial \mathbb{D}$ and $0<r<2$. In the following proof, we will find a positive constant $C^{*}$ which does not depend on $\xi$ and $r$ such that

$$
\iint_{B(\xi, r) \cap \mathbb{D}}\left|S_{\mathrm{H}}\left(f_{2}\right)\right|^{2}\left(1-|z|^{2}\right)^{3} d x d y \leq C^{*} r .
$$

We first consider one special case: there exists $g \in G$ such that $g(B(\xi, r) \cap \mathbb{D}) \subset \mathscr{F}$. By Lemma 2, we know that $\left|S_{\mathrm{H}}\left(f_{2}\right)\right|^{2}\left(1-|z|^{2}\right)^{3} d x d y$ is a Carleson measure on the domain $g(B(\xi, r) \cap \mathbb{D})$. Then, we have

$$
\begin{aligned}
& \iint_{B(\xi, r) \cap \mathbb{D}}\left|S_{\mathrm{H}}\left(f_{2}(w)\right)\right|^{2}\left(1-|w|^{2}\right)^{3} d u d v \\
& \quad=\iint_{g(B(\xi, r) \cap \mathbb{D})}\left|S_{\mathrm{H}}\left(f_{2} \circ g^{-1}(z)\right)\right|^{2}\left(1-\left|g^{-1}(z)\right|^{2}\right)^{3}\left|\left(g^{-1}\right)^{\prime}(z)\right|^{2} d x d y \\
& \quad=\iint_{g(B(\xi, r) \cap \mathbb{D})}\left|S_{\mathrm{H}}\left(f_{2} \circ g^{-1}(z)\right)\right|^{2}\left(1-|z|^{2}\right)^{3}\left|\left(g^{-1}\right)^{\prime}(z)\right|^{5} d x d y \\
& \quad=\iint_{g(B(\xi(\xi) \cap) \cap \mathbb{D})}\left|S_{\mathrm{H}}\left(f_{2}(z)\right)\right|^{2}\left(1-|z|^{2}\right)^{3}\left|\left(g^{-1}\right)^{\prime}(z)\right|^{5} d x d y \\
& \quad=\iint_{g(B(\xi, r) \cap \mathbb{D})}\left|S_{\mathrm{H}}\left(f_{2} \circ g^{-1}(z)\right)\right|^{2}\left(1-|z|^{2}\right)^{3}\left|\left(g^{-1}\right)^{\prime}(z)\right| d x d y \\
& \quad=\iint_{g(B(\xi, r) \cap \mathbb{D})}\left|S_{\mathrm{H}}\left(f_{2}(z)\right)\right|^{2}\left(1-|z|^{2}\right)^{3}\left|\left(g^{-1}\right)^{\prime}(z)\right| d x d y .
\end{aligned}
$$

The second above equality holds since

$$
\frac{\left|g^{\prime}(z)\right|}{1-|g(z)|^{2}}=\frac{1}{1-|z|^{2}} \quad \text { for any } g \in G \text {. }
$$

Since $g$ is a Möbius transformation $g(B(\xi, r) \cap \mathbb{D})$ is a chord-arc domain, from Lemma 4 , we have

$$
\begin{aligned}
& \iint_{g(B(\xi, r) \cap \mathbb{D})}\left|S_{\mathrm{H}}\left(f_{2}(z)\right)\right|^{2}\left(1-|z|^{2}\right)^{3}\left|\left(g^{-1}\right)^{\prime}(z)\right| d x d y \\
& \quad \leq C_{5} \int_{\partial g(B(\xi, r) \cap \mathbb{D})}\left|\left(g^{-1}\right)^{\prime}(z)\right| d s=C_{5} \int_{\partial(B(\xi, r) \cap \mathbb{D})} d s \leq 2 \pi C_{5} r,
\end{aligned}
$$

where $C_{5}$ depends only on the the Carleson norm. Hence, we have

$$
\iint_{B(\xi, r) \cap \mathbb{D}}\left|S_{\mathrm{H}}\left(f_{2}(w)\right)\right|^{2}\left(1-|w|^{2}\right)^{3} d u d v \leq 2 \pi C_{5} r .
$$

For any $1 \leq i \leq n$, since $B_{i} \cap \partial \mathbb{D}$ contains no limit points of $G$ and there are finitely many $g_{1}, \cdots, g_{m}$ belonging to $G$ such that

$$
\left(B_{i} \cap \mathbb{D}\right) \subset \bigcup_{1}^{m} g_{j}(\mathscr{F})
$$

then we can get that the measure $\left|S_{\mathrm{H}}\left(f_{2}(z)\right)\right|^{2}\left(1-|z|^{2}\right)^{3} d$ $x d y$ is a Carleson measure on $B_{i} \cap \mathbb{D}$.

Now, we consider the general case. Let $G^{*}$ be the set of all the elements $g$ in $G$ such that $g(B) \cap B(\xi, r) \neq \varnothing$. When $g \in G^{*}$, there are at most three possibilities as follows:

(a) There exists $1 \leq i \leq n, g\left(B_{i} \cap \mathscr{F}\right) \subset B(\xi, r)$

(b) There exists $1 \leq i \leq n, g\left(B_{i}\right) \cap B(\xi, r) \neq \varnothing$ and $g\left(I_{i}\right)$ $\subset B(\xi, r) \cap \partial \mathbb{D}$

(c) There exists $1 \leq i \leq n, g\left(B_{i}\right) \cap B(\xi, r) \neq \varnothing$ and $g\left(I_{i}\right)$ $\cap B(\xi, r) \cap \partial \mathbb{D} \neq \varnothing$

In Case (a), we have

$$
\begin{aligned}
& \iint_{g\left(B_{i} \cap \mathscr{F}\right)}\left|S_{\mathrm{H}}\left(f_{2}(w)\right)\right|^{2}\left(1-|w|^{2}\right)^{3} d u d v \\
& \quad \leq \iint_{g\left(B_{i} \cap \mathbb{D}\right)}\left|S_{\mathrm{H}}\left(f_{2}(w)\right)\right|^{2}\left(1-|w|^{2}\right)^{3} d u d v \\
& \quad=\left.\iint_{B_{i} \cap \mathbb{D}}\left|S_{\mathrm{H}}\left(f_{2} \circ g(z)\right)\right|^{2}\left(1-|g(z)|^{2}\right)^{3}|| g^{\prime}(z)\right|^{2} d x d y \\
& \quad=\left.\iint_{B_{i} \cap \mathbb{D}}\left|S_{\mathrm{H}}\left(f_{2} \circ g(z)\right)\right|^{2}\left(1-|z|^{2}\right)^{3}|| g^{\prime}(z)\right|^{5} d x d y \\
& \quad=\left.\iint_{B_{i} \cap \mathbb{D}}\left|S_{\mathrm{H}}\left(f_{2}(z)\right)\right|^{2}\left(1-|z|^{2}\right)^{3}|| g^{\prime}(z)\right|^{5} d x d y \\
& \quad=\iint_{B_{i} \cap \mathbb{D}}\left|S_{\mathrm{H}}\left(f_{2} \circ g(z)\right)\right|^{2}\left(1-|z|^{2}\right)^{3}|| g^{\prime}(z) \mid d x d y \\
& \quad=\iint_{B_{i} \cap \mathbb{D}}\left|S_{\mathrm{H}}\left(f_{2}(z)\right)\right|^{2}\left(1-|z|^{2}\right)^{3}|| g^{\prime}(z) \mid d x d y \\
& \quad \leq C_{5} \int_{\partial\left(B_{i} \cap \mathbb{D}\right)}\left|g^{\prime}(z)\right| d s \\
& \quad=C_{5} \int_{\partial g\left(B_{i} \cap \mathbb{D}\right)} d s \leq \pi C_{5} \text { length }\left(g\left(B_{i} \cap \partial \mathbb{D}\right)\right),
\end{aligned}
$$

where the second above inequality holds by Lemma 4 and $C_{5}$ only depends on the Carleson norm of $\left|S_{\mathrm{H}}\left(f_{2}(z)\right)\right|^{2}$ $\left(1-|z|^{2}\right)^{3} d x d y$ on $B_{i} \cap \mathbb{D}$. 
For Case (b), we have

$$
\begin{aligned}
& \iint_{g\left(B_{i} \cap \mathscr{F}\right) \cap B(\xi, r)}\left|S_{\mathrm{H}}\left(f_{2}(w)\right)\right|^{2}\left(1-|w|^{2}\right)^{3} d u d v \\
& \quad \leq \iint_{g\left(B_{i} \cap \mathbb{D}\right) \cap B(\xi, r)}\left|S_{H}\left(f_{2}(w)\right)\right|^{2}\left(1-|w|^{2}\right)^{3} d u d v \\
& \quad \leq \pi C_{5} \operatorname{length}\left(B_{i} \cap \partial \mathbb{D}\right) .
\end{aligned}
$$

For Case (c), notice that $g\left(B_{i} \cap \mathbb{D}\right) \cap B(\xi, r)$ is a triangle with three circle arcs and the angle corresponding to the side $g\left(B_{i} \cap \partial \mathbb{D}\right) \cap B(\xi, r)$ is bigger than some constant. Thus, we have

length $\left(\partial\left(g\left(B_{i} \cap \mathbb{D}\right) \cap B(\xi, r)\right)\right) \leq C_{6}$ length $\left(g\left(B_{i} \cap \partial \mathbb{D}\right) \cap B(\xi, r)\right)$,

where $C_{6}$ depends on the Carleson norm of $\left|S_{\mathrm{H}}\left(f_{2}\right)\right|^{2}$ $\left(1-|z|^{2}\right)^{3} d x d y$ on $B_{i} \cap \mathbb{D}$ and the angle between $\partial B_{i}$ and $\partial \mathbb{D}$.

Similar to Case (a), we have

$$
\begin{aligned}
& \iint_{g\left(B_{i} \cap \mathscr{F}\right) \cap B(\xi, r)}\left|S_{\mathrm{H}}\left(f_{2}(w)\right)\right|^{2}\left(1-|w|^{2}\right)^{3} d u d v \\
& \leq \pi C_{6} \text { length }\left(g\left(B_{i} \cap \partial \mathbb{D}\right) \cap B(\xi, r)\right) .
\end{aligned}
$$

For any $1 \leq i \leq n$, the arc $B_{i} \cap \partial \mathbb{D}$ does not contain the limit points of $G$. Therefore, for any $g_{1}, g_{2} \in G^{*}$, if $g_{1}\left(B_{i}\right) \cap$ $B(\xi, r) \neq \varnothing$ and $g_{2}\left(B_{i}\right) \cap B(\xi, r) \neq \varnothing$, then the images of $B_{i}$ $\cap \partial \mathbb{D}$ under $g_{1}, g_{2}$ do not overlap. Thus, we have

$$
\begin{aligned}
& \iint_{B(\xi, r) \cap \mathbb{D}}\left|S_{\mathrm{H}}\left(f_{2}(w)\right)\right|^{2}\left(1-|w|^{2}\right)^{3} d u d v \\
& \quad \leq \pi C^{*} \sum_{g \in G^{*}} \operatorname{length}(g(B) \cap B(\xi, r) \cap \partial \mathbb{D}) \\
& \quad \leq \pi C^{*} \operatorname{length}(B(\xi, r) \cap \partial \mathbb{D}) \leq 2 \pi^{2} C^{*} r,
\end{aligned}
$$

where $C^{*}$ equals to the maximum value of the constants, appearing in the proof of this theorem, and $B=\bigcup_{i}^{n}\left(B_{i} \cap \mathbb{D}\right)$. The proof of this theorem is complete.

Theorem 6. Let $G$ be a finitely generated Fuchsian group of the second kind with some parabolic elements and $\mathscr{F}$ be the Dirichlet fundamental domain of $G$ centered at 0 . For a $G$ -compatible univalent harmonic function $f$, if there exists a constant $C>0$ such that, for any $\xi \in \mathscr{F}(\infty)$ and any $0<r<2$,

$$
\iint_{B(\xi, r)}\left|S_{H}(f)\right|^{2}\left(1-|z|^{2}\right)^{3} \chi_{\mathscr{F}} d x d y \leq C r,
$$

then $\left|S_{H}(f)\right|^{2}\left(1-|z|^{2}\right)^{3} d x d y$ is in $C M(\mathbb{D})$, where $\chi_{\mathscr{F}}$ is the characteristic function of the Dirichlet fundamental domain $\mathscr{F}$.

Proof. Let $G$ be a finitely generated Fuchsian group of second kind with some parabolic elements. Without loss of generality, suppose that the generator of $G$ contains only one parabolic element $\gamma$ and $\xi \in \mathscr{F}(\infty)$ is one fixed point of $\gamma$.
Similar to the proof of Theorem 5, we divide $f$ into two parts $f_{1}, f_{2}$ as follows:

$$
f_{1}=\sum_{g \in G} f \chi_{g\left(\mathscr{F}^{*}\right)}, f_{2}=\sum_{g \in G} f \chi_{g(B \cap \mathscr{F})} .
$$

where $\mathscr{F}^{*}=\mathscr{F}-(B \cap \mathscr{F})$ and $B$ is a sufficiently small disk with center $\xi$ and radius $r_{0}$ such that $\partial B$ intersects with the sides of $\mathscr{F}$ having $\xi$ as a common vertex. Let $\gamma_{0}$ be the arc of $\partial B$ between the sides of $\mathscr{F}$ which have $\xi$ as a common vertex.

By Theorem 5, we know that $\left|S_{\mathrm{H}}\left(f_{1}(z)\right)\right|^{2}\left(1-|z|^{2}\right)^{3} d x d$ $y \in \mathrm{CM}(\mathbb{D})$. Then, we only need to show that $\left|S_{\mathrm{H}}\left(f_{2}(z)\right)\right|^{2}$ $\left(1-|z|^{2}\right)^{3} d x d y$ is a Carleson measure in $\mathbb{D}$.

For any $g \in G$, we have

$$
\begin{aligned}
& \iint_{\mathcal{g}(B \cap \mathscr{F})}\left|S_{\mathrm{H}}\left(f_{2}(w)\right)\right|^{2}\left(1-|w|^{2}\right)^{3} d u d v \\
& \quad \leq \iint_{g(B \cap \mathscr{F})} \frac{C_{1}}{1-|w|^{2}} d u d v=\iint_{B \cap \mathscr{F}} \frac{C_{1}\left|g^{\prime}(z)\right|^{2}}{1-|g(z)|^{2}} d x d y \\
& \quad=\iint_{B \cap \mathscr{F}} \frac{C_{1}\left|g^{\prime}(z)\right|}{1-|z|^{2}} d x d y=C_{1} \iint_{B \cap \mathscr{F}} \frac{1-|g(z)|^{2}}{\left(1-|z|^{2}\right)^{2}} d x d y \\
& \quad \leq C_{2}\left(1-\left|g\left(z_{0}\right)\right|^{2}\right) \iint_{B \cap \mathscr{F}} \frac{1}{\left(1-|z|^{2}\right)^{2}} d x d y \leq C\left(1-\left|g\left(z_{0}\right)\right|^{2}\right),
\end{aligned}
$$

where $z_{0}$ is any point in $\gamma_{0}$. The first above inequality holds by Theorem 5 of [1], and the second and final above equalities hold by

$$
\frac{\left|g^{\prime}(z)\right|}{1-|g(z)|^{2}}=\frac{1}{1-|z|^{2}} \quad \text { for any } g \in G
$$

In the second above inequality, the hyperbolic length of $\gamma_{0}$ is finite, where $C_{2}$ depends on $z_{0}$ and the hyperbolic length of $\gamma_{0}$. In the final inequality, the finiteness of the hyperbolic area of $B \cap \mathscr{F}$ is given by Theorem 1.2 in Huo [7].

Let $\eta$ be any point in $\mathbb{D}$ and $0<r<1$. By the proof of Lemma 2.2 in Huo [7], we know that the sequence $\left\{g\left(z_{0}\right)\right\}_{g \in G}$ is an interpolating sequence. Therefore, we obtain that

$$
\sum_{g \in G}\left(1-\left|g\left(z_{0}\right)\right|^{2}\right) \delta_{g\left(z_{0}\right)} \in \mathrm{CM}(\mathbb{D}),
$$

where $\delta_{z}$ stands for the Dirac mass at $z$. Thus, the images of $B \cap \mathscr{F}$ under $g$, contained in the disk $B(\eta, r)$, satisfy

$$
\begin{aligned}
& \iint_{B \cap \mathbb{D}}\left|S_{\mathrm{H}}\left(f_{2}(w)\right)\right|^{2}\left(1-|w|^{2}\right)^{3} d u d v \\
& \quad \leq \sum_{g \in G, g(B \cap \mathscr{F}) \cap B(\eta, r) \neq \varnothing} \iint_{g(B \cap \mathscr{F})}\left|S_{\mathrm{H}}\left(f_{2}(w)\right)\right|^{2}\left(1-|w|^{2}\right)^{3} d u d v \leq C r .
\end{aligned}
$$

Thus, the proof of the theorem is complete. 
Proof of Theorem 1. Combining Theorem 5 and Theorem 6, the proof of Theorem 1 is complete.

\section{Data Availability}

The (data type) data used to support the findings of this study are included within the article.

\section{Conflicts of Interest}

The authors declare that they have no conflicts of interest.

\section{Acknowledgments}

This work was supported the National Natural Science Foundation of China Grant Number 11871085 and PhD research startup foundation of Jinling Institute of Technology Grant Numbers jit-b-202011, jit-b-202049, and jit-fhxm-2018.

\section{References}

[1] R. Hernández and M. J. Martín, "Pre-Schwarzian and Schwarzian derivatives of harmonic mappings," Journal of Geometric Analysis, vol. 25, no. 1, pp. 64-91, 2015.

[2] M. Chuaqui, P. Duren, and B. Osgood, "Ellipses, near ellipses, and harmonic Möbius transformations," Proceedings of the American Mathematical Society, vol. 133, no. 9, pp. 27052710, 2005.

[3] M. Chuaqui, P. Duren, and B. Osgood, "Univalence criteria for lifts of harmonic mappings to minimal surfaces," Journal of Geometric Analysis, vol. 17, no. 1, pp. 49-74, 2007.

[4] M. Chuaqui, P. Duren, and B. Osgood, "Schwarzian derivative criteria for valence of analytic and harmonic mappings," Mathematical Proceedings of the Cambridge Philosophical Society, vol. 143, no. 2, pp. 473-486, 2007.

[5] F. Dal'Bo, Geodesic and Horocyclic Trajectories, Springer, 2011.

[6] J. B. Garnett, Bounded Analytic Functions, Academic Press, New York, 1981.

[7] S. Huo, "On Carleson measures induced by Beltrami coefficients being compatible with Fuchsian groups," Annales Academiae Scientiarum Fennicae Mathematica, vol. 46, 2021.

[8] A. F. Beardon, The Geometry of Discrete Group, SpringerVerlag, 1983.

[9] M. Zinsmeister, "Les domaines de Carleson," The Michigan Mathematical Journal, vol. 36, no. 2, pp. 213-220, 1989. 